\title{
Dementia in Indigenous Populations
}

\author{
Kylie Catania and Peter K Panegyres*
}

Neurodegenerative Disorders Research Pvt Ltd, West Perth, Australia

\section{Introduction to Research on Dementia}

Only recently have we begun to recognise the importance of research into dementia in low and middle-income countries and in ethnic minorities, including immigrant and indigenous groups, within high income countries. The neurological research community has started to consider that ethnicity itself, as well as environmental factors unique to individual communities, may influence the onset and rates of dementia. Our recent paper reviewed data on 6,500 subjects with Alzheimer's Disease (AD) from a multinational clinical trials data repository. We reported that the odds of Early Onset AD in the USA were significantly higher for African Americans and Native Alaskans, Americans and Hawaiians, after controlling for co-morbidities and gender [1]. A commentary by the same authors will further discuss factors related to ethnicity and dementia for ethnic minorities in North America [2]. This commentary will be a brief summary focussing on dementia in low-income countries and in Indigenous populations, outside of North America. According to the World Health Organisation (WHO) there is no official definition of the term 'indigenous', but it can include groups who have strong links to territories, historical continuity with precolonial societies, that form non-dominant groups in society and have distinct social, economic or political systems [3]. There are almost 400 million indigenous people worldwide and their health outcomes are generally much poorer compared to non-indigenous people $[3,4]$. The prevalence of dementia is rapidly increasing and none more so than in low income and Indigenous populations.

\section{Epidemiological studies in Africa}

Epidemiological studies thus far have been unable to accurately estimate the prevalence of dementia in Africa. A 2014 review found prevalence rates of $2.29 \%$ to $21.6 \%$ [5]. Most of the studies reviewed reported a lower prevalence of dementia compared to developed countries; however a few of the most recent studies reported prevalence rates similar to Western countries [5]. This may be due to newer, more accurate methods of assessment or the evolution of the disease in Africa as the population becomes exposed to greater risk factors, or, most likely, a combination of the two. The authors discussed some of the inherent difficulties in measuring prevalence as that many of the studies to date have been in single communities, which may not be representative of the specific country and that the variety of languages and cultures that exist between and within countries make assessment of cognition difficult [5]. When examining dementia and health more broadly and from the perspective of indigenous studies, we may need to take a different perspective and assess and address the needs of individual groups within a country on their own merits, rather than attempting to develop a one-size-fits-all approach, which is preferred in more culturally homogenous Western countries.

One study has highlighted the difference in prevalence reports based on the tools used to test for dementia [6]. In rural Tanzania, researchers compared the dementia diagnosis rates using the 10/66 Dementia Research Group tool and the DSM-IV criteria. The 10/66 tool was designed for use in the developed world and low literacy populations. Prevalence rates were 3.38 times higher when using the 10/66 criteria compared with the DSM-IV. The authors raised some pertinent points for this discrepancy, such as, that prodromal or mild dementia may be missed by the DSM-IV due to the focus on the functional impact of the impairment. Occupations may be more physical and less cognitively demanding and the supportive and protective unit of the family may help to compensate for mild impairments. Conversely, the 10/66 tool may over estimate prevalence, by identifying people with depression or other mental health disorders as having dementia. These issues highlight the complex difficulties in assessing for dementia in culturally diverse communities.

Low educational attainment has consistently been reported as a risk factor for the development of dementia in high income countries and is considered one of seven modifiable risk factors for Alzheimer's Disease, with a Population Attributable Risk (PAR) of 19.1\% [7]. The association between education level and dementia has been less consistent in research emerging from Africa. A study conducted with the Chagga and Maasai peoples of rural Tanzania measured educational attainment as a surrogate for cognitive reserve and found no association between the clinical diagnosis of dementia and educational attainment [8]. The authors surmised that in a population with very limited access to formal education, years of education were less likely to be a good marker of cognitive reserve, with implications for the assessment of cognition in these populations and preventative programs for dementia. Unique methods of assessment and treatment for dementia specific to the requirements of individual communities, are required. In Tanzania, interviews with traditional healers and Christian faith healers revealed pervasive beliefs that dementia was a normal part of the aging process and there was no name for it [9]. Some people in the community believed that their memory loss was a result of bewitching, which could therefore be stigmatising. The Tanzanian research group has begun to work with local healers to help identify people with dementia and to help educate the community [9]. They have also implemented a small feasibility study to look at the use of Cognitive Stimulation Therapy as a culturally appropriate and economically viable treatment option, with promising results [10].

\section{Epidemiological studies in Asia}

The Asian nations of China, India, Japan and Indonesia are in the top 7 countries that have the largest number of people with dementia [11]. They have large numbers of ethnic minorities, in which dementia is unstudied. A 2017 systematic review and meta-analysis demonstrated that more than $60 \%$ of people with dementia have not been diagnosed and that the rates of undetected dementia were highest in India and China, when compared with Europe and North America [12]. APOE

*Corresponding author: Professor PK Panegyres, Neurodegenerative Disorders Research Pvt Ltd, 4 Lawrence Avenue, West Perth, Australia, Tel: +61 89481 6293; Fax: +61 89481 6294; E-mail: research@ndr.org.aul

Received August 28, 2017; Accepted August 29, 2017; Published August 31, 2017

Citation: Catania K, Panegyres PK (2017) Dementia in Indigenous Populations. J Neurol Disord 5: 362. doi:10.4172/2329-6895.1000362

Copyright: @ 2017 Catania K, et al. This is an open-access article distributed under the terms of the Creative Commons Attribution License, which permits unrestricted use, distribution, and reproduction in any medium, provided the original author and source are credited. 
$\varepsilon 4$, an allele associated with an increased risk of $\mathrm{AD}$, has demonstrated some variability in risk across ethnicities. Our commentary in press [2] discusses the variability in APOE for ethnicities in North America. A 2010 study [13] concluded that the presence of APOE $\varepsilon 4$ was a risk factor for late-onset $A D$ in Mongolian carriers $(\mathrm{OR}=2.23)$. Another study [14], reported APOE $\varepsilon 4$ as a much higher risk factor for the Azeri Turkish community in Iran, with Odds Ratios between 6.6 and 17.8 depending on model of inheritance.

A very low prevalence of $\mathrm{AD}$ of $1.33 \%$ has been reported in the rural, high altitude Tibetan population from Qinghai, China [15]. The authors postulated that this may be partly due to a low life expectancy (69.96 years). Read meat consumption and head injury were found to be associated with AD. Meditative practices, an important component of Tibetan culture, was found to be protective. The authors highlighted that the Tibetan population have lived at high altitudes longer than any other ethnic group, altering their physiology, however the effect of altitude on $\mathrm{AD}$ was not investigated directly and may be an interesting avenue of future research.

\section{Epidemiological studies in Oceania}

A small body of evidence now exists on dementia in indigenous Australians, in both rural and remote communities and those living in cities and regional areas. Studies are repeatedly reporting much higher rates of dementia in Aboriginal Australians, compared with nonIndigenous Australians, with rates amongst the highest in the world [16-18]. A longitudinal study of indigenous people living in rural and remote areas of the Kimberley, Western Australia had a dementia prevalence of $14.8 \%$ [16] and a study of indigenous people living in urban and regional New South Wales found a prevalence of $13.4 \%$, agestandardised to $21 \%$, compared with the overall Australian prevalence of $6.8 \%$ [17]. The Aboriginal population has an overwhelming burden of poor health outcomes, cardiovascular risk factors, drug and alcohol abuse, educational, employment and social hardships [19]. Most researchers working with these communities highlight the complexity of delineating the risk factors that are contributing to dementia for Aboriginal people. A 2010 study identified older age and male gender as the non-modifiable factors associated with dementia. Lack of formal schooling, head injury, smoking, stroke and epilepsy were the modifiable factors associated with high rates of dementia for indigenous Australians [20]. The above mentioned longitudinal study found an association between stroke, head injury, analgesic medication, low $\mathrm{BMI}$ and high blood pressure and the decline from normal cognition to cognitive impairment or dementia [16].

Australia has produced one of the only published, validated cognitive and dementia assessment tool designed specifically for an indigenous population [21]. The Kimberley Indigenous Cognitive Assessment Tool (KICA) was developed in consultation with linguists, health workers and community elders, for use in rural and remote Western Australia [22]. The authors had identified the inherent weaknesses of using the available assessment tools with this population, such as limited or no access to formal education, indigenous languages being generally oral and differing concepts of number, time and space. Based on a small body of data, recent systematic review has since supported the use of the KICA for Aboriginal Australians outside of the Kimberley, when other available tests are not considered appropriate [23].

\section{Epidemiological studies in South America}

A 2014 study [24] described an overall prevalence of dementia of $4.9 \%$ in a Brazilian Amazonian Indigenous population that had a mean age of 62.3 years. The population was unique in that it was highly homogenous, significantly geographically isolated and researchers could sample over $50 \%$ of the total population. Almost all participants had little to no formal schooling and the cardiovascular risk factors of cholesterol levels and blood pressure were extremely low. Other dementia risk factors in the population were not measured. A poster presentation [25] on a separate Brazilian Amazonian population described a prevalence of $6.4 \%$ with a mean age of participants of 72.4 years, reported to be lower than the prevalence in non-Indigenous Brazilians. Authors in both studies postulated that lower cardiovascular risk was protective for these communities, despite low educational attainment, however the second study reported not insignificant rates of smoking and alcoholism. A Colombian study [26] attempted to estimate the ancestry of a group of individuals with late onset $\mathrm{AD}$ compared to controls using genotyping and found that a Native American ancestry was associated with a statistically significantly lower risk of AD. Factors such as age, sex, APOE status, education and socioeconomic status were controlled, but not vascular risk factors. Whether Native American ethnicity is protective for the development of sporadic $\mathrm{AD}$ requires further research.

\section{Epidemiological studies in Europe}

A 2013 paper from the Nursing literature [27] highlighted the impact of culture on the experience of dementia and the effective care of patients. In this work, interviews were performed with 15 family members and staff of a nursing home, 13 of whom were Sami, an Indigenous group from Northern Scandinavia and North-West Russia. The themes that emerged were the importance of a common language, a culturally familiar environment, diet, continuing spiritual practices and rhythms of life. For example, the large, high-ceilinged communal living room contributed to distress for some patients, but traditional lavvo (tents) in the outdoor areas were comforting. As patients with dementia progressively lose their identity through their loss of memory and independence, it is imperative to avoid accommodating such individuals in culturally strange housing. As the number of indigenous peoples with dementia grows, it is important to understand their culture-related needs, as well the more 'scientific' aspects of dementia.

\section{Conclusion}

We have provided a brief snap shot of some of the important research emerging on dementia in indigenous societies - an area of research which was previously sparse and thought to be unnecessary. Common themes have emerged across continents. These include, the complexity of intersecting modifiable and non-modifiable risk factors, difficulties in obtaining accurate statistics, barriers to early diagnosis, such as suitable cognitive assessments for the population that are language and culturally appropriate, lack of community education, acceptance of dementia as part of indigenous culture and reluctance to diagnose a condition for which support and effective treatment is lacking. It can be extremely difficult for researchers foreign to a culture, to design an appropriate study for that population. Studies completed within indigenous groups or low-income countries may not be published in peer-reviewed journals, not have sufficient scientific rigour or be available in English. It is clear to researchers and clinicians that dementia is an emerging pandemic in our aging populations, however there are many parts of the world with populations for whom there exists no data on dementia. We did not find any published research on dementia in Indigenous groups in middle to high income countries such as Maori in New Zealand or Adivasis in India. There remain a large number of low income countries across Africa, Asia, South America and Oceania for whom there is no research on dementia at all. There is a pressing need for further work in this field and we look forward to contributing to and learning from future studies. 


\section{References}

1. Chen HY, Panegyres PK (2016) The role of ethnicity in Alzheimer's Disease: Findings from the C-PATH online data repository. J Alzheimers Dis 51: 515-523.

2. Chen HY, Panegyres PK (2017) Ethnic differences in early onset Alzheimer's Disease. J Alzheimers Dis Parkinsonism 7: 1-4.

3. World Health Organization (2007) Health of indigenous peoples. [online] Available at: http://www.who.int/mediacentre/factsheets/fs326/en/ [Accessed 3 Aug. 2017].

4. Flicker L, LoGiudice $D$ (2015) What can we learn about dementia from research in Indigenous populations? Int Psychogeriatr 27: 1957-1958.

5. Olayinka OO, Mbuyi NN (2014) Epidemiology of dementia among the elderly in Sub-Saharan Africa. Int J Alzheimers Dis 20: 1-15.

6. Paddick SM, Longdon A, Kisoli A, Dotchin C, Gray WK, et al. (2013) Dementia prevalence estimates in sub-Saharan Africa: comparison of two diagnostic criteria. Glob Health Action 6: 1-7.

7. Norton S, Matthews FE, Barnes DE, Yaffe K, Brayne C (2014) Potential for primary prevention of Alzheimer's disease: an analysis of population-based data. Lancet Neurol 13: 788-794.

8. Paddick SM, Longdon A, Gray WK, Dotchin C, Kisoli A, et al. (2014) The association between educational level and dementia in rural Tanzania. Dement Neuropsychol 8: 117-125.

9. Hindley G, Kissima J, Oates LL, Paddick SM, Kisoli A, et al. (2016) The role of traditional and faith healers in the treatment of dementia in Tanzania and the potential for collaboration with allopathic healthcare services. Age and Ageing 46:130-137.

10. Paddick SM, Mkenda S, Mbowe G, Kisoli A, Gray WK, et al. (2017) Cognitive stimulation therapy as a sustainable intervention for dementia in sub-Saharan Africa: Feasibility and clinical efficacy using a stepped-wedge design. Int Psychogeriatr 29: 979-989.

11. Catindig JAS, Venketasubramanian N, Ikram MK, Chen C (2012) Epidemiology of dementia in Asia: Insights on prevalence, trends and novel risk factors. J Neurol Sci 321: 11-16.

12. Lang L, Clifford A, Wei L, Zhang D, Leung D, et al. (2017) Prevalence and determinants of undetected dementia in the community: A systematic literature review and meta-analysis. BMJ Open 7: e011146.

13. Huriletemuer, Wang B, Wang J, Wang G, Zhang C, et al. (2010) APOE $\varepsilon 4$ is a high-risk factor for Alzheimer's disease in the Mongolian population. J Neurol Sci 288: 167-169.

14. Rezazadeh M, Khorrami A, Yeghaneh T, Talebi M, Jalal-Kiani S, et al. (2016)
Genetic factors affecting late-onset Alzheimer's Disease susceptibility. Neuromolecular Med 18:37-49.

15. Huang F, Shang Y, Luo Y, Wu P, Huang X, et al. (2016) Lower prevalence of Alzheimer's Disease among Tibetans: Association with religious and genetic factors. J Alzheimers Dis 50:659-667.

16. Lo Giudice D, Smith K, Fenner S, Hyde Z, Atkinson D, et al. (2016) Incidence and predictors of cognitive impairment and dementia in Aboriginal Australians: A follow-up study of 5 years. Alzheimers Dement 12: 252-261.

17. Radford K, Mack HA, Draper B, Chalkley S, Daylight G, et al. (2015) Prevalence of dementia in urban and regional Aboriginal Australians. Alzheimers Dement 11: $271-279$.

18. Li SQ, Guthridge SL, Aratchige PE, Lowe MP, Wang Z, et al. Dementia prevalence and incidence among the Indigenous and non-Indigenous populations of the Northern Territory. Med J Aust 200: 465-469.

19. AlHW (2011) The health and welfare of Australia's Aboriginal and Torres Strait Islander people: an overview 2011. Cat. no. IHW 42: 127

20. Smith K, Flicker L, Dwyer A, Atkinson D, Almeida OP, et al. (2010) Factors associated with dementia in Aboriginal Australians. Aust N Z J Psychiatry 44: 888-893.

21. Warren LA, Shi Q, Young K, Borenstein A, Martiniuk A (2015) Prevalence and incidence of dementia among Indigenous populations: a systematic review. Int Psychogeriatr 27: 1959-1970.

22. LoGiudice D, Smith K, Thomas J, Lautenschlager NT, Almeida OP, et al. (2006) Kimberley Indigenous Cognitive Assessment tool (KICA): development of a cognitive assessment tool for older indigenous Australians. Int Psychogeriatr 18: $269-280$.

23. Dyer SM, Laver K, Friel M, Whitehead C, Crotty M (2017) The diagnostic accuracy of the Kimberley Indigenous Cognitive Assessment (KICA) too: A systematic review. Australas Psychiatry 25: 282-287.

24. Dozzi Brucki SM, Nitrini R (2014) Cognitive impairment in individuals with low educational level and homogenous sociocultural background. Dement Neuropsychol 8: 345-350.

25. Caixeta L (2011) Dementia prevalence in an indigenous population from Brazilian Amazon. Alzheimers Dement 7: S604.

26. Moreno DJ, Pino S, Rios A, Lopera F, Ostos H, et al. (2017) Genetic ancestry and susceptibility to late-onset Alzheimer's Disease (LOAD) in the admixed Colombian population. Alzheimer Dis Assoc Disord 31: 225-231.

27. Hanssen I (2013) The influence of cultural background in intercultural dementia care: exemplified by Sami patients. Scand J Caring Sci 27: 231-237. 